\title{
DISEÑO Y APLICACIÓN DE UNA ESCALA DE ACTITUDES HACIA EL ESTUDIO DE LAS CIENCIAS EXPERIMENTALES
}

\author{
ORTEGA RUIZ, P., ${ }^{1}$ SAURA SOLER, J.P., ${ }^{2}$ MÍNGUEZ VALLEJOS , R., ${ }^{3}$, GARCÍA DE LAS BAYONAS \\ CAVAS, A. ${ }^{4}$ y MARTÍNEZ MARTÍNEZ,,${ }^{5}$ \\ 'Departamento de Teoría e Historia de la Educación. Universidad de Murcia. \\ ${ }^{2}$ Inspector Técnico de Educación. \\ ${ }^{3}$ Departamento de Teoría e Historia de la Educación. Universidad de Murcia. \\ - Departamento de Física y Química del IFP Almirante Bastarreche. Cartagena. \\ ${ }^{5}$ Departamento de Física y Química del IFP Torre Pacheco. Cartagena.
}

\section{SUMMARY}

This work presents a design for a scale of attitudes when studying Experimental Sciences. We describe the process of elaboration and its application in two secondary schools, as well as the analysis of the obtained results.

\section{INTRODUCCIÓN}

Al interés trađicional por transmitir los conocimientos científicos se ha unido en los últimos años una preocilpación creciente por los métodos de enseñanza-aprendizaje (Novak 1982,1987, Osborne y Vitrock 1983,1986, Driver 1983,1986,1988, Posner 1982, Gil 1983 1985,1986). La razón de ello estriba, por un lado, en el escaso interés que los estudiantes muestran por el aprendizaje de las ciencias (Gauld 1982,1980, Schibeci 1984) y el bajo rendimiento escolar que obtienen en estas disciplinas (Krajkovich 1982, Munby 1983, Oliver 1988, Simpson 1990, Schibeci 1986,1989 ) por otro, en la probada incidencia que los métodos, como variable mediacional, ejercen en los procesos de enseñanza-aprendizaje (Hasan 1985, Saura $1988,1989,1990,1991)$.

En la práctica didáctica el dominio cognitivo acapara toda la atención y esfuerzo. Ello ha llevado a que se primen fundamentalmente las estrategias de enseñanza enfocadas a la adquisición de conocimientos; por el contrario, el ámbito afectivo, especialmente la formación de actitudes positivas hacia el estudio, ha quedado tradicionalmente relegado. El profesor, orientador del proceso de enseñanza-aprendizaje (Ortega 1986, p. 66), vive más alejado del dominio afectivo que de la comprensión de conceptos y procesos y del desarrollo de destrezas en el ámbito cognitivo (Escudero 1985). Las razones del abandono del ámbito afectivo no radican, en buena medida, sólo en el profesorađo: los problemas existentes sobre el concepto (Ortega 1983) y la dificultad en la evaluación de las actitudes (Escámez 1986), junto a la presión de la sociedad y de la propia Administración por los aprendizajes cognitivos estarían en la raíz del mismo.

A nuestro juicio, la enseñanza de las ciencias debería prestar más atención al ámbito afectivo, ya que a nadie se le escapa la incidencia cada vez mayor que tiene esta dimensión educativa en todos los procesos de enseñanza-aprendizaje (Hasan 1985). De hecho, aunque en nuestro país son pocos los trabajos sobre las actitudes relacionadas con la ciencia (Escudero y Lacasta 1984, Escudero 1985 , Moreno 1987, Arana 1985,1987, Serrano 1986,1988, Gil 1987, Moreno 1987, Gómez 1989, Solbes 1989, Espinosa 1991), es patente la abundante literatura que hay en otros países (fundamentalmente anglosajones); prueba de ello son las revisiones bibliográficas realizadas por Fraser (1977), Gadner (1983), Schibeci (1984), Saura (1991), que se centran fundamentalmente en la búsqueda de correlaciones estadísticas entre las actitudes y otras variables de interés didáctico, como el ren. dimiento escolar, el clima o ambiente de clase, el uso de 
materiales curriculares innovadores, diferentes estrategias didácticas, etc.

El objetivo de este trabajo es diseñar una escala que nos permita conocer las actitudes de nuestros estudiantes hacia el estudio de las Ciencias Experimentales. Para ello, comenzamos describiendo los diferentes procedimientos de construcción de escalas de actitudes y, más adeiante, nos centramos en el proceso de construcción de nuestra escala. Por último, describimos la aplicación de la misma y el análisis de los resultados obtenidos.

\section{LAS ESCALAS DE ACTITUDES}

No es éste el lugar para hacer una larga exposición sobre la teoría đe Ias actitudes, los procesos de su formación y cambio y la problemática de su medida. Nos basta con exponer el concepto de actitud del que aquí se parte, y que es hoy ampliamente compartido por la mayoría de los autores. Para nosotros una actitud es «una disposición aprendida para responder consistentemente de un modo favorable o desfavorable a un objeto social dado», siendo, de este modo, el elemento informativo (ideas, creencias), la aceptacion-rechazo y la conducta sus componentes esenciales. A juicio de Fishbein y Ajzen (1980) y otros muchos autores, el elemento afectivo (aceptación-rechazo) constituye su componente más radical, y aquello que lo distingue de otros constructos más próximos (hábito, opinión, rasgo, valor, etc.)

Por lo que se refiere al campo específico de la medición de actitudes existen, en general, muchas y serias dificultades que los especialistas exponen con todo detalie (Dawes 1975, Padua 1979, Rodríguez 1989). Hasta ahora se han consolidado por su uso los instrumentos elaborados por Thurstone $(1928,1929)$, Likert (1932), Guttman (1944), Osgood (1957), Triandis (1974). En la actualidad, Ias preferencias se inclinan por el procedimiento Likert, ya que permite obtener con relativa facilidad escalas de actitudes con altos índices de validez y fiabilidad.

En el campo específico de las ciencias se han desarrollado y aplicadodiversos instrumentos de exploración; ejemplos de ellos son el «Inventario de Actitudes Científicas», de Moore y Sutman (1970), que utiliza el procedimiento Likert; el «Test de Actitudes Rejativas a la Ciencia», de Fraser (1978), también por el procedimiento Likert; la "Escala de Actitudes Científicas», de Billeh y Zakhariades (1975), que utiliza el procedimiento Thurstone; el «Test sobre Actitudes Científicas», de Kozlow y Nay (1976); el «Inventario de Intereses Científicos», de Stevens y Atwood (1978), que utiliza la técnica del diferencial semántico; etc.

A pesar de las numerosas escalas que se han diseñado y la amplia variedad de aspectos que se contemplan en todas ellas, nos hemos visto obligados a diseñar nuestra propia escala. Por un lado, todos los cuestionarios se han diser̃ado en otros países en los que, por razones obvias, los ambientes socio-culturales de los estudiantes son muy diferentes al nuestro; este aspecto, como se sabe, es un factor que influye decisivamente en la elaboración de una escaia y que obliga a revisarias cuando se apiican en contextos diferentes a los originales. Por otro lado, la población escolar sobre la que vamos a actuar (alumnos de Formación Profesional) tiene características singula * res, muy diferentes a las de sus homólogos de otros niveles educativos, lo que obliga a matizar el tipo de cuestiones que se presentan en la escala. Por último, el objeto de actitud que vamos a medir no ha sido tratado específicamente por los investigadores.

Cono es bien sabido las actitudes no son realidades empíricas directamente observables. Inferimos su existencia de las manifestaciones, verbales o escritas, de los sujetos estudiados. La medida de la actitud, por lo tanto, se ve condicionada por la naturaleza de su objeto. De aquí que necesariamente acudamos a cuestionarios o autoinformes para constatar la presencia, signo e intensidad de una determinada actitud en los sujetos en estudio.

En general, las escalas de actitudes están elaboradas para medir sólo el componente afectivo; es decir, la aceptación-rechazo del sujeto hacia el objeto de actitud, así como su intensidad. La medida del componente cognitivo y conductual de la actitud ha tenido, hasta ahora, menos interés.

Una escala de actitudes consiste en una serie de proposiciones (ítems) relativas a la aceptación o rechazo de un objeto. El número de ítems debe ser lo suficientemente grande para poder abarcar todas las posibles facetas del objeto y de la actitud hacia él, ya que las actitudes no son algo simple sino que pueden tener múltiples dimensio. nes. Además, una buena escala de actitudes debe ser fiable y válida; es decir, todos los ítems referidos a una misma actitud deben estar correlacionados entre sí (consistencia interna) y deben ser instrumentos estables de medición (utilizables en más de una ocasión y en situaciones análogas).

\section{EL PROCESO DE ELABORACIÓN DE LA ESCALA}

Para elaborar la escala de actitudes hacia el estudio de las ciencias experimentales hemos elegido el procedimiento Likert, ya que goza de gran aceptación entre los investigadores por su mayor objetividad y por la relativa facilidad con que permite obtener escalas aceptablemente fiables. La construcción de escalas por este método exige recoger un gran número de ítems medianamente favorables/desfavorables (los ítems extremosos son di* fícilmente aceptables por los encuestados) que, a juicio del investigador, estén relacionados con la actitud que se va a medir.

Para la formulación fiteral de los ítems se deben tener en cuenta los criterios enunciados por Edward (1957) y la distribución de éstos se debe hacer al azar, cuidando que aproximadamente la mitad de ellos presenten una actitud positiva y la otra mitad negativa. Por último, si operamos con escalas de cinco grados de intensidad en el continuo aceptación-rechazo o acuerdo-desacuerdo, asignamos el 
valor 5 a «muy de acuerdo o muy favorable», 4 a «de acuerdo», 3 a «indiferente», 2 a «en desacuerdo» y 1 a «muy en desacuerdo o muy desfavorable».

Para llegar a la escala definitiva se realiza el tratamiento estadístico adecuado de cada ítem, seleccionando aquéllos que tengan un alto poder de discriminación y que midan la misma actitud, que son los que constituirán la escala definitiva.

Para elaborar nuestra escala seleccionamos 85 ítems que hacían referencia a aspectos relativos al estudio de las Ciencias Experimentales, a la importancia de estas asignaturas, a las clases y actividades extraescolares relacionadas con ellas, a los textos y otros medios de divulga-

Tabja I

Muestra los valores de la validez de construcción de cada uno de los ftems que componen la escala.

\begin{tabular}{lllll|} 
P1 & 0.592 & P2 & 0.581 \\
P3 & 0.603 & P4 & 0.682 \\
P5 & 0.587 & P6 & 0.564 \\
P7 & 0.579 & P8 & 0.645 \\
P9 & 0.706 & P10 & 0.679 \\
P11 & 0.675 & & P12 & 0.679 \\
P13 & 0.473 & & \\
\hline
\end{tabular}

Tabla II

Muestra los valores obtenidos sobre la fiabilidad de la escala de actitudes.

\begin{tabular}{|c|c|c|c|c|}
\hline \multicolumn{5}{|c|}{ TEST SCORE STATISTIC } \\
\hline & TOTAL & TOT/14 & ODD & EVEN \\
\hline MEAN & 45.886 & 3.278 & 22.801 & 23.085 \\
\hline STD DEV & 9.076 & 0.648 & 4.604 & 4.980 \\
\hline STD ERR & 0.626 & 0.045 & 0.318 & 0.344 \\
\hline MAXIMUM & 169.000 & 4.929 & 35.000 & 34.000 \\
\hline MINIMUM & 21.000 & 1.500 & 11.000 & 10.000 \\
\hline N. CASES & 211 & 211 & 211 & 211 \\
\hline \multicolumn{5}{|c|}{ INTERNAL CONSISTENCY DATA } \\
\hline \multicolumn{3}{|c|}{ SPLIT-HALF CORRELATHON } & \multicolumn{2}{|l|}{0.794} \\
\hline \multicolumn{3}{|c|}{ SPEARMAN-BROWN COEFFICIENT } & \multicolumn{2}{|l|}{0.885} \\
\hline \multicolumn{3}{|c|}{ GUTTMAN (RULON) COEFFICIENT } & 0.883 & \\
\hline \multicolumn{3}{|c|}{ COEFFICIENT ALPHA-ALL ITEMS } & 0.866 & \\
\hline \multicolumn{3}{|c|}{ COEFFICIENT ALPHA-ODD ITEMS } & 0.743 & \\
\hline \multicolumn{3}{|c|}{ COEFFICIENT ALPHA-EVEN ITEMS } & 0.768 & \\
\hline
\end{tabular}

ción científica, etc. Los ítems confeccionados se presentaron a «expertos» para que seleccionaran aquéllos que, cumpliendo con las condiciones de Edward (1957), se consideraran más relacionados con el objeto de actitud propuesto.

El cuestionario provisional fue aplicado a una muestra de 211 alumnos, seleccionados al azar, de cuatro institutos de Formación Profesional de características similares a aquéllos a los que se aplicaría después el cuestionario definitivo. Cuidamos especialmente que los alumnos tuvieran las mismas edades (14-16 años) y estudiaran las mismas ramas profesionales.

El cuestionario definitivo, que se muestra en el anexo I, consta de 13 ítems y los resultados obtenidos sobre la validez de construcción de la escala se muestran en las tablas 1 y 2 . Considerando una probabilidad mayor o igual a 0,45 la tabla I nos indica que todos los ítems son adecuados para el objeto de actitud propuesto. Los valores de fiabilidad se muestran en la tabla II; podemos observar que el coeficiente SPEARMAN-BROWN y el coeficiente ALPHA-ALL ÍTEMS (coeficiente de CRONBACH) tienen valores muy próximos a la unidad, lo que pone de manifiesto la alta fíabilidad de la escala elaborada.

\section{RESULTADOS OBTENIDOS Y DISCUSIÓN}

La aplicación de la escala definitiva se ha realizado en dos institutos de Formación Profesional de la provincia de Murcia. En el instituto de FP Torre Pacheco se seleccionó un grupo de tercer nivel de la rama Administrativa y Comercial con 33 alumnos (grupo A) y otro de segundo nivel de la misma rama con 24 alumnos (grupo B). En el instituto de FP Almirante Bastarreche, de Cartagena, se seleccionaron dos grupos de segundo nivel de la rama Peluquería y Estética con 29 alumnos el grupo A y 25 alumnos el grupo $B$.

Antes de aplicar la escala, debido a que nuestros estudiantes noestán acostumbrados a responder a este tipo de cuestiones, les explicamos con detalle el contenido de cada ítem y su sentido positivo o nevativo, tomando todas las precauciones para no influir en el sentido de sus respuestas; además, les dimos todo el tiempo necesario para evitar, en la medida de lo posible, los errores accidentales que pudieran cometer en su contestación.

Las tablas III y IV (Anexo II) muestran para cada ítem el número total de respuestas dadas al cuestionario por ios alumnos de los grupos A y B del instituto de FP de Cartagena. Se muestran también las puntuaciones totales por ítem en valor absoluto y porcentual. Las tablas $V$ y VI (Anexo II) muestran los mismos resultados para los grupos A y B del instituto de FP Torre Pacheco.

Los resultados obtenidos por los grupos A y B del IFP Torre Pacheco se muestran en las figuras 1 y 2 . La figura 1 recoge de forma comparada los resultados globales de la actitud de cada grupo; los valores de $-20 \%$ y $-25 \%$ nos 
Figura I

Representa la actitud global de los grupos A y B del IFP Torre Pacheco en valor porcentual.

\section{ACTITUDES HACIA EL ESTUDIO DE LAS CIENCIAS EXPERIMENTALES \\ IFP TORRE PACHECO}

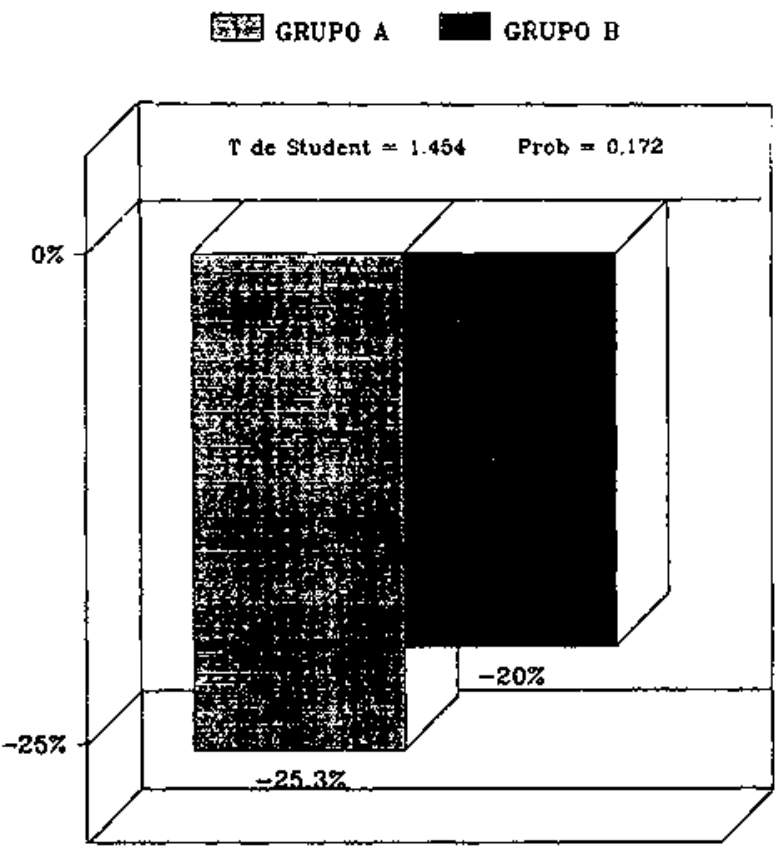

indican que la actitud de los alumnos hacia el estudio de las Ciencias Experimentales es marcadamente negativa; además, éstos son muy similares y el análisis de la varianza nos da un valor para la T de Student de 1,454 para una probabilidad de 0,172 , lo que nos indica que no hay diferencias estadísticamente significativas entre ambos grupos; es decir, son muy similares en cuanto al objeto de actitud medido.

La figura 2 muestra de forma comparada los resultados globales de la actitud de cada grupo en cada uno de los ítems. Se observa que, excepto en los ítems 7 y 13, la actitud de ambos grupos es marcadamente negativa. Estos ítems son globalmente valorados de forma positiva por los alumnos; es decir, conceden cierta utilidad a estas asignaturas y están moderadamente atentos en clase.

Los ítems valorados más negativamente son el 5,8 y 12 ; de su análisis se infiere que los estudiantes no consideran útil el estudio de las Ciencias para su formación profesional, le dan poca importancia a estas asignaturas y, por supuesto, su estudio no les produce ninguna satisfacción. Cabe destacar, por último, el valor negativo de los ítems 1 y 10 ; de su análisis se desprende que a estos estudiantes les resulta desagradable el estudio de estas disciplinas y las clases, aunque de forma moderada, les resultan aburridas y pesadas.
Figura 2

Representa la actitud global en cada ítem de los grupos A y B de] IFP Torre Pacheco en valor porcentual.

\section{ACTITUDES HACIA EL ESTUDIO DE LAS \\ CIENCIAS EXPERIMENTALES}

IFP TORRE PACHECO

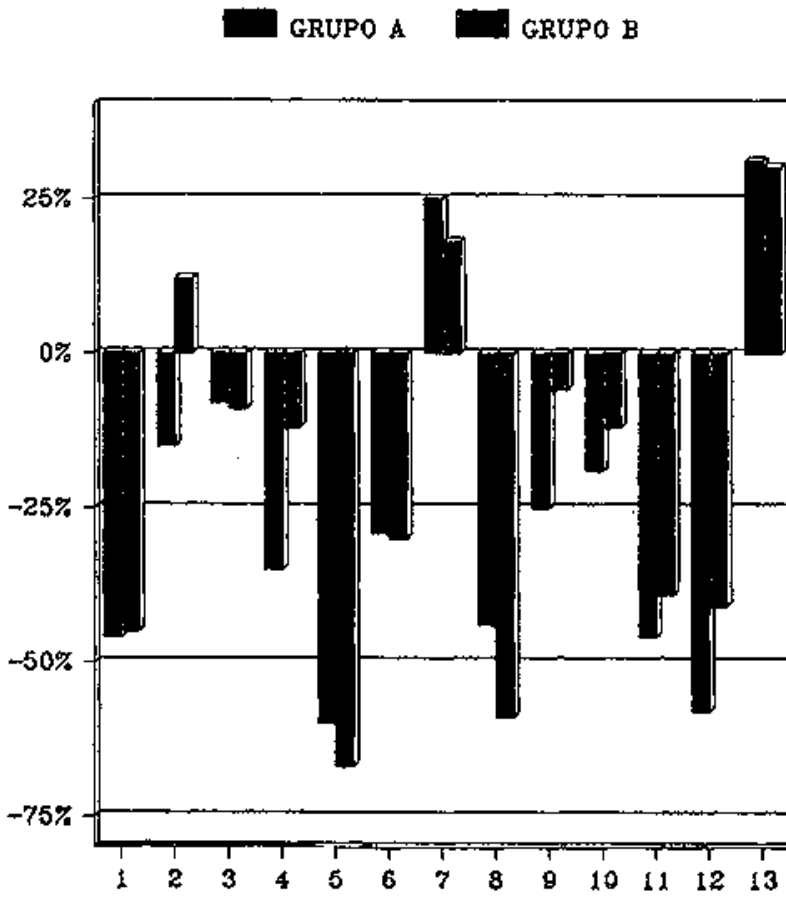

Los resultados obtenidos por los grupos A y B del IFP de Cartagena se muestran en las figuras 3 y 4 . Estos resultados son similares a los encontrados en el otro centro. Los valores de $-5 \%$ y $-14 \%$ nos confirman la actitud negativa de ambos grupos y el valor de Ia varianza ( $T$ de Student) de 2,616 para una probabilidad de 0,023 nos indica que tampoco hay diferencias estadísticamente significativas entre ambos.

En un análisis ítem a ítem destaca el valor positivo de los f́tems 7 y 13; es decir, al igual que ocurría en el otro instituto, los estudiantes conceden cierta utilidad a estas asignaturas y están moderadamente atentos en clase. Para el grupo A el resto de los ítems es, en mayor o menor proporción, negativo; sin embargo, en el grupo B hay varios ítems que son, de forma moderada, valorados positivamente. Del análisis de ellos se infiere que a los alumnos de este grupo les resulta menos pesado el estudio de estas asignaturas, les gusta más resolver problemas, encuentran más interesante todo lo relacionado con las Ciencias y las clases se les hacen menos aburridas y pesadas que a los del otro grupo.

Por último, como conclusión final, del análisis global de los datos obtenidos en ambos centros, se desprende que las asignaturas de Ciencias Experimentales no son del agrado de nuestros estudiantes y su estudio les resulta poco agradable y bastante difícil y aburrido. Además, las 
Figura 3

Representa la actitud global de los grupos A y 8 del IFP de Cartagena en valor porcentual.

\author{
ACTITUDES HACIA EL ESTUDIO DE LAS \\ CIENCIAS EXPERIMENTALES \\ IFP ALMIRANTE BASTARRECHE
}

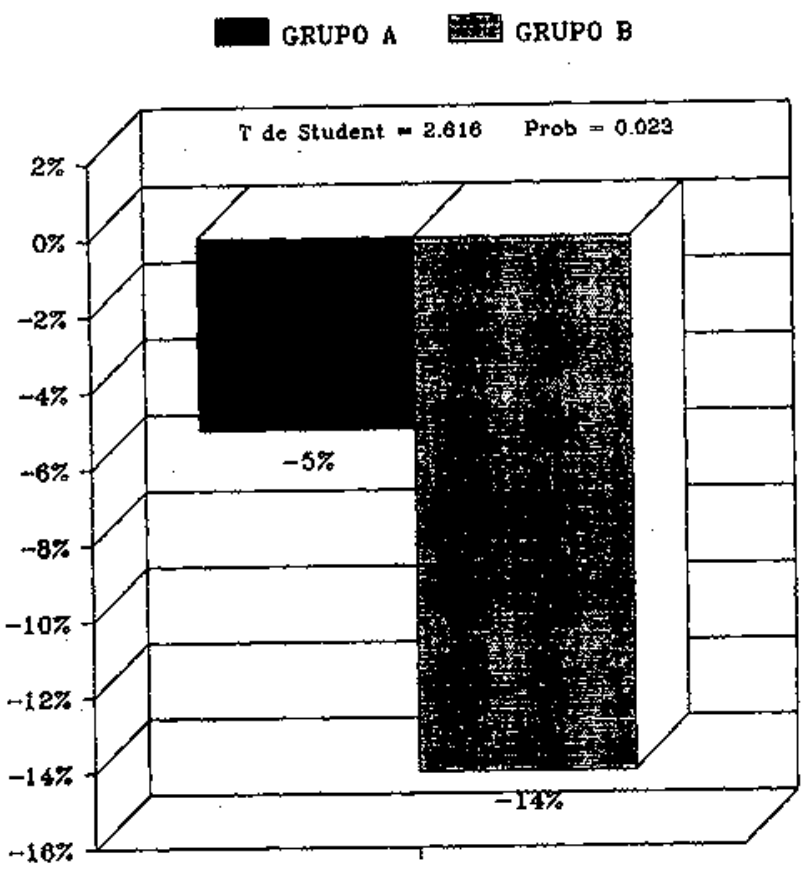

consideran poco importantes en las enseñanzas de For* mación Profesional y poco útiles para su futuro profesional.

Si está demostrado suficientemente que la variable actitud juega un papel importante en el rendimiento acadé.

\section{REFERENCIAS BIBLIOGRÁFICAS}

ARANA, J., ESCUDERO, T., GARCÉS, R. y PALACIOS, E., 1985. Imagen de las asignaturas de ciencias en la transición de la educación básica a la secundaria, Enseñanza de las Ciencias, Vol, extra, p. 20.

ARANA, y., ESCUDERO, T., GARCÉS, R. y PALACIAN, E., 1987. Imagen de las asignaturas de ciencias en la transición de la educación básica a la secundaria, Enseñanza de las Ciencias, 5(1), pp, 10-15.
Figura 4

Representa la actitud global en cada ítem de los grupos A y B de! IFP de Cartagenà en valor porcentual.

\section{ACTITUDES HACIA EL ESTUDIO DE LAS \\ CIENCIAS EXPERIMENTALES \\ IFP ALMIRANTE BASTARRECHE}

GRUPO A GRUPO. B

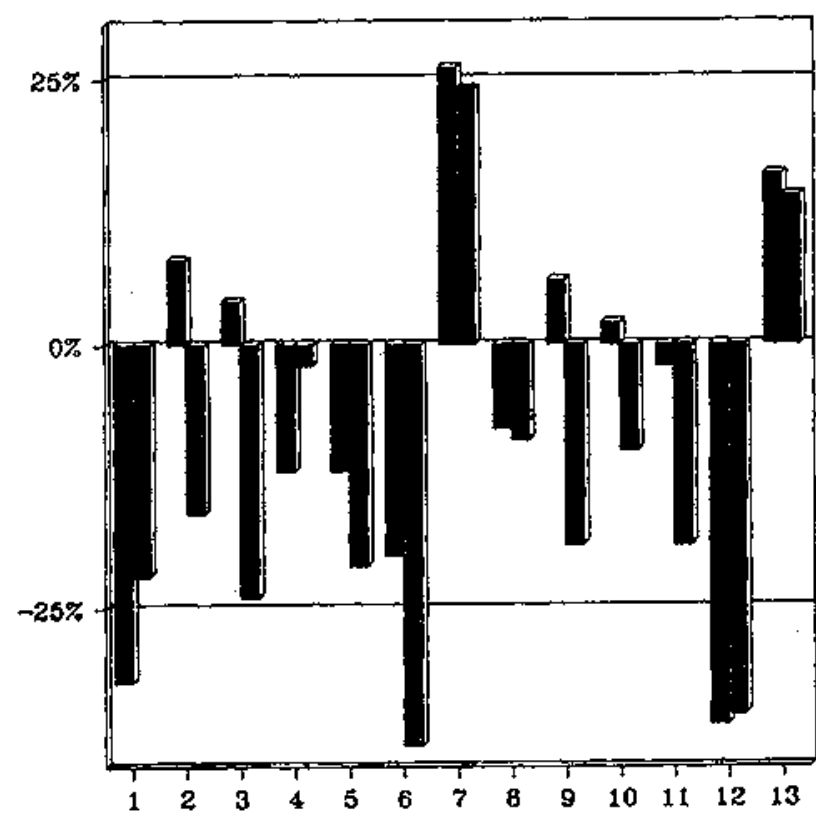

mico de los alumnos, el estudio de dicha variable se hace del todo imprescindible. La escala que aquí se ofrece intenta ser una aportación en tai sentido. Su uso, obviamente, ha de hacerse con las debidas cautelas en otros contextos que no presenten análogas características socio-culturales de la población escolar aquí estudiada.
BILLEH, V.Y. y ZAKHARLADES, G.A., 1975. The development and application of a scale for measuring scientific attitudes, Science Education, 59, pp. 155-165.

DAWES, R.M., 1975. Fundamentos y técnicas de medición de actitudes. (Limusa: México).

DRIVER, R., 1983. Theories-in-Action: some theoretical and empirical issues in the study of student's conceptual 
frameworks in science, Studies in Science Education, 10 , pp. 37-70.

DRIVER, R., 1986. Psicología cognitiva y esquemas conceptuales de los alumnos, Enseñanza de las Ciencias, 6 (2), pp. 109-120.

DRIVER, R, 1988. Un enfoque constructivista para el desarrolio del curriculum, Enseñanza de las Ciencias, 6 (2), pp. 109 . 121.

EDWARDS, A.L., 1957. Techniques of attitude scale construction. (New York).

ESCÁMEZ, J., 1986. La educación en actitudes y valores: una exigencia para el hombre de hoy, en Escámez y Ottega, La Enseñanza de Actitudes y Valores, pp, 126-129. (Nau Llibres: Valencia).

ESCUDERO, T., 1985. Las actitudes en la enseñanza de las ciencias: un panorama complejo, Revista de Educación, 278, pp. 5-26.

ESCUDERO, T y LACASTA, E, 1984, Las actitudes científi* cas de los futuros maestros en relación con stus conocimien* tos, Enseñanza de las Ciencias, 2(3), pp. 175-180.

ESPINOSA, J, y ROMÁN, T., 1991. Actitudes hacia la ciencia y asignaturas pendientes: dos factores que afectan al rendimiento en ciencia, Enseñanza de las Ciencias, 9(2), pp. 151155.

FRASER, B.J., 1977. Selection and validation of attitude scales for curriculum evaluation, Science Education, 61 , pp. 317 319.

FRASER, B.J., 1978. Development of a test of science -related attitudes, Science Education, 62, pp. 509-515.

GADNER, P.L., 1983. Interest in science and technology education: an Australian perspective. Paper for presentation to the International Symposium Institute for Science Education. University of Kiel. Federal Republic of Germany. April, pp. 2-6.

GAULD, C., 1982. The scientific attitude and science education: a critical reappraisal, Science Education, 66, pp. $109-$ 129.

GLL, D., 1983. Tres paradigmas básicos en la enseñanza de las ciencias, Enseñanza de las Ciencias, 1, pp. 26-33.

GIL, D., 1985. El futuro de la enseñanza de las ciencias, Revista de Educación, 178, pp. 27-38.

GIL, D., I986. La metodología científica y la enseñanza de las ciencias unas relaciones controvertidas, Enseñanza de las Ciencias, $4(2)$, pp. $111-121$.

GIL, D. MARTÍNEZ, J. y SENET, F., 1987. Actitudes y expectativas del profesorado ante la resolución de problemas de Física. Ponencia presentada al II Congreso Internacional sobre investigación en la didáctica de las ciencias y de las matemáticas, Enseñanza de las Ciencias, Vol. extra, pp. 203. 205 .

GÓMEZ, C. y CERVERA, S., 1989. Actitudes y cambio conceptual en la educacion ambiental. Ponencia presentada al II Congreso Internacional sobre la Didactica de las Ciencias y de las Matemáticas, Enseñanza de las Ciencias, Vol. extra, pp. 209-211.
GUTTMAN, L., 1944. A basic for scaling quantitative data, Am. Social. Rev, 9, pp. $139-150$.

HASAN, O.E., 1985. An investigation into factors affecting attitudes toward science of secondary school students in Jordan, Science Education, 69(1), pp, 3-19.

KOZLOW, M.J. y NAY, M.A., 1976. An approach to measuring scientific attitudes, Science Education, 60 , pp. 147-162.

KRAJKOVICH, J.G. y SMITH., 1982. The development of the image of science and scientists scale, Journal of Research in Science Teaching, 19, pp. 39-44.

LIKERT, R., 1932. A technique for the measurement of attitude, Archives of Phychology, 140, pp. 44-45.

MOORE, R.H. y SUTMAN, F.X., 1970. The deveiopment field test, and validation of an inventory of scientific attitudes, Journal of Research in Science Teaching, 7, pp. 85-94.

MORENO, M.D. y GIL, D., 1987. La medida de las actitudes de los estudiantes de BUP hacia la Física. Ponencia presentada al Segundo Congreso Internacional de la Didáctica de las Ciencias y de las Matemáticas, Enseñanza de las Ciencias, Vol. extra, pp. 268-270.

MUMBY, M., 1983. Thirty studies involving the scientific attitude inventory: what confidence can we have in the instrument?, Journal of Research in Science Teaching, 20, pp.141-162.

NOVAK, J., 1982. Teoría y práctica de la educación. (Alianza: Madrid).

NOVAK, J., 1987. Human constructivism: Towards a unity of phychological and epistemological meaning making. (Comunicación, Ithaca: New York, julio 1987).

OLIVER, J.S. y SIMPSON, R.D., 1988. Influences of attitude toward science, achievement motivation, and science self concept on achievement in science: a longitudinal study, Science Education. 72(2), pp. 143-157.

OSBORNE y VITTROCK, 1983, Learning Science: a generative process, Science Education, 67, pp. 490-508.

OSBORNE y VITTROCK, 1985. The generative leaming model and its implications for science education, Studies in Science Education,12, pp. 59-87.

ORTEGA, P., 1986a. Estrategias para el cambio de actitudes I. Dimensiones prácticas de los modelos de Fishbein, Ajzen y Bandura, en Escámez y Ortega, La enseñanza de actitudes y valores. (Nau Llibres: Valencia).

ORTEGA, P., 1986b. La investigación en la formación de actitudes: problemas metodológicos y conceptuales, Anales de Pedagogía, 4, Universidad de Murcia, p. 15.

OSGOOD, C., SUCI, G. Y TANNENBAUN, P., 1975. The measurement of learning. (Urbana Univ. III Press.).

PADUA, J., 1979. Técnicas de investigación aplicadas a las Ciencias Sociales. (FCE: México), pp. 180-194.

POSNER et al., 1982. Accommodation of a scientific conception: towards a theory of conceptual change, Science Education, 66, pp. $211+227$.

RODRÍGUEZ, A. y SEOANE, J., 1989. Creencias, actitudes y 
valores, (Alhambra Universidad: Madrid).

SAURA, I.P., GARCIA, A. y MARTÍNEZ, F, 1988. Metodología activa de una experiencia física, Apuntes de Educación, 31 octubre-diciembre, pp. 4-6

SAURA,J.P., GARCIA, A. y MARTÍNEZ, F., 1989. Cinemática de una esfera por un plano inclinado, Apuntes de Educacion, 33 abril-junio, pp. 12-15.

SAURA, J.P. y GARCÍA, A., 1990. Fuerza y potencia de un juguete, Comunidad Escolar, 18, p. 15.

SAURA, J.P., 1991. La formación de actitudes positivas hacia el estudio de las Ciencias Experimentales, Tesis doctoral. Universidad de Murcia.

SCHIBECI, R.A., 1984. Attitudes to science: an update, Studies in Science Education, 12, pp. 25-57.

SCHIBECI, R.A., 1989. Home, school, and peer group. Inftuences on student attitudes and achievement, Science Education, 73, pp. 13-25.

SCHIBECI, R.A. y RILEY, J.P., 1986. Influence of students background and perceptions on science attitudes and achievernent, Journal of Research in Science Teaching, 23(3), pp. $177-189$.
SERRANO, T., 1986. La imagen de los científicos en los alumnos al finalizar el Ciclo Medio, Documentos IEPS, Monografías, 1.

SERRANO, T., 1988. Actitudes de los alumnos y aprendizaje de las ciencias, un estudio longitudinal, Investigación en la Escuela, 5, pp. 29-38.

SIMSON, R.D. y OLIVER, J.S., 1990. A summary of major influences on attitudes toward an achievement in science among adolescent students, Science Education, 74, pp. 1-19.

SOLBES, I. y VILCHES, A., 1989. Interacciones cienciatécnica-sociedad: un instrumento de cambio actitudinal, Enseñanza de las Ciencias, 7(1), pp. 14-20.

STEVENS, J.T. y ATWOOD, R.K., 1978. Interest scores as predictors of science process performance for junior high students, Science Education, 62, pp. 303-308.

THURSTONE, L., 1928. Attitudes can be measured, Am. Jour Phychol. 33, pp. 529-554.

THURSTONE, L., 1929. Theory of attitude measurement, Phychol. Rev., 36, pp. 222-241.

TRIANDIS, H.L., 1974. Actitudes y cambio de actitudes, (Toray: Barcelona).

\section{ANEXO I}

\section{ESCALA}

ITEM N 1: El estudio de las Ciencias Experimentales es el que con más agrado realizo 12345

ITEM N ${ }^{2}$ : $\mathrm{El}_{1}$ estudio de las Ciencias Experimentales me resulta algo pesado porque no le veo utilidad 12345

ÍTEM N $N^{9}$ 3: Me gusta resolver problemas relacionados con las Ciencias Experimentales 12345

ITEM N²: Me resulta desagradable estudiar las asignaturas de Ciencias Experimentales 12345

ITEM No 5: Me interesa el estudio de las Ciencias Experimentales porque lo considero importante como preparacion para encontrar un puesto de trabajo 2345

ITEM $N^{9}$ 6: No me gustan las asignaturas de Ciencias Experimentales porque su estudio me resulta difícil 12345

ITEM N ${ }^{2}$ : Las asignaturas de Ciencias Experimentales s6́lo sirven para suspender y obtener malas notas . 12345
ÍTEM No 8: Considero que las asignaturas de Ciencias Experimentales deberían tener más importancia en las enseñanzas de Formación Profesional 12345

ÍTEM No 9: Todo to relacionado con las Ciencias Experimentales lo encuentro interesante 12345

ÍTEM N 10: Lasclases de Ciencias Experimentales se me hacen aburridas y pesadas 12345

ÍTEM N 11: Las asignaturas de Ciencias Experimentales las considero importantes porque me ayudan a reflextonar mejor para mi futura profesión 12345

ITEMN $^{2}$ 12: El estudio de las Ciencias Experimentales me produce satisfaccion 12345

ITEM N 13: Normalmente «desconecto» en la clase de Ciencias Experimentales 12345 


\section{ANEXO II}

Tabla III

Resultados obtenidos por el grupo A del instituto de FP de Cartagena después de la aplicación de la escala de actitudes hacia el estudio de las Ciencias Experimentales.

\begin{tabular}{|cccccccccc|}
\hline ÍTEM & $N^{2}$ DE 1 & $N^{2}$ DE 2 & $N^{2}$ DE 3 & $N^{2}$ DE 4 & $N^{2}$ DE 5 & TOTAL & $\%$ & TOTAL (+0.) & $\%$ \\
\hline 1 & 6 & 7 & 11 & 4 & 1 & 74 & 39 & -13 & -22 \\
2 & 6 & 7 & 6 & 10 & 0 & 78 & 42 & -9 & -16 \\
3 & 8 & 8 & 6 & 4 & 3 & 73 & 38 & -14 & -24 \\
4 & 5 & 3 & 12 & 6 & 3 & 86 & 49 & -1 & -2 \\
5 & 7 & 4 & 12 & 6 & 0 & 75 & 40 & -12 & -21 \\
6 & 13 & 6 & 3 & 7 & 0 & 63 & 29 & -22 & -38 \\
7 & 2 & 2 & 6 & 18 & 1 & 101 & 62 & +14 & +24 \\
8 & 3 & 5 & 16 & 4 & 1 & 82 & 46 & -5 & -9 \\
9 & 5 & 9 & 7 & 8 & 0 & 76 & 41 & -11 & -19 \\
10 & 6 & 6 & 5 & 12 & 0 & 81 & 45 & -6 & -10 \\
11 & 7 & 5 & 10 & 6 & 1 & 76 & 41 & -11 & -19 \\
12 & 8 & 7 & 11 & 3 & 0 & 67 & 33 & -20 & -35 \\
13 & 3 & 1 & 13 & 9 & 3 & 95 & 57 & +8 & +14 \\
TOTAL & 79 & 70 & 118 & 97 & 13 & 1027 & 43 & -102 & -14 \\
\hline
\end{tabular}

Tabla IV

Resultados obtenidos por el grupo B del instituto de FP de Cartagena después de la aplicación de la escala de actitudes hacia el estudio de las Ciencias Experimentales.

\begin{tabular}{|ccccccccccc|}
\hline ITEM & $N^{2}$ DE 1 & $N^{2}$ DE 2 & $N^{2}$ DE 3 & No DE 4 & $N^{2}$ DE 5 & TOTAL & $\%$ & TOTAL (+o $)$ & $\%$ \\
\hline 1 & 4 & 11 & 8 & 1 & 1 & 59 & 34 & -16 & -32 \\
2 & 2 & 6 & 4 & 12 & I & 79 & 54 & +4 & +8 \\
3 & 3 & 5 & 8 & 5 & 4 & 77 & 52 & +2 & +4 \\
4 & 5 & 5 & 6 & 9 & 0 & 69 & 44 & -6 & -12 \\
5 & 2 & 9 & 8 & 5 & 1 & 69 & 44 & -6 & -12 \\
6 & 4 & 10 & 4 & 6 & 1 & 65 & 40 & -10 & -20 \\
7 & 4 & 1 & 3 & 10 & 7 & 90 & 65 & +13 & +26 \\
8 & 6 & 3 & 6 & 9 & 1 & 71 & 46 & -4 & -8 \\
9 & 3 & 3 & 10 & 6 & 3 & 78 & 53 & +3 & +6 \\
10 & 4 & 4 & 4 & 13 & 0 & 76 & 51 & +1 & +2 \\
11 & 4 & 3 & 10 & 6 & 2 & 74 & 49 & -1 & -2 \\
12 & 5 & 10 & 8 & 2 & 0 & 57 & 32 & -18 & -36 \\
13 & 2 & 5 & 4 & 11 & 3 & 83 & 58 & +8 & +16 \\
TOTAL & 48 & 75 & 83 & 95 & 24 & 948 & 48 & -30 & -5 \\
\hline
\end{tabular}


Tabla $\mathrm{V}$

Resultados obtenidos por el grupo A del instituto de FP Torre Pacheco después de la aplicación de la escala de actitudes hacia el estudio de las Ciencias Experimentales.

\begin{tabular}{|c|c|c|c|c|c|c|c|c|c|}
\hline ITEM & $\mathrm{N}=\mathrm{DE} \mathrm{i}$ & $\mathrm{N}^{2} \mathrm{DE} 2$ & № DE 3 & $\mathrm{~N}^{2} \mathrm{DE} 4$ & $\mathrm{~N}^{2} \mathrm{DE} 5$ & TOTAL & $\%$ & TOTAL $(+0-)$ & $\%$ \\
\hline 1 & 11 & 9 & 12 & 1 & 0 & 69 & 27 & -30 & -46 \\
\hline 2 & 3 & 5 & 7 & 17 & 1 & 107 & 56 & +8 & +12 \\
\hline 4 & 6 & 5 & 12 & 9 & 1 & 93 & 46 & -6 & -9 \\
\hline 5 & 4 & 9 & 11 & 9 & 0 & 91 & 44 & -8 & .12 \\
\hline 6 & 5 & 16 & 6 & 6 & 0 & 79 & 35 & -20 & -30 \\
\hline 7 & 0 & 8 & 10 & 10 & 5 & 111 & 59 & +12 & +18 \\
\hline 8 & 13 & 14 & 5 & 1 & 0 & 60 & 21 & -39 & -59 \\
\hline 9 & 2 & 7 & 18 & 5 & 1 & 95 & 47 & -4 & -6 \\
\hline 10 & 4 & 7 & 15 & 7 & 0 & 91 & 44 & -8 & -12 \\
\hline$\$ 1$ & 6 & 16 & 9 & 2 & 0 & 73 & 30 & -26 & -39 \\
\hline 12 & 9 & 10 & 13 & 1 & 0 & 72 & 30 & -27 & -41 \\
\hline 13 & 0 & 2 & 12 & 16 & 3 & 119 & 65 & +20 & +30 \\
\hline TOTAL & 78 & 122 & 134 & 84 & 11 & 1115 & 40 & -172 & -20 \\
\hline
\end{tabular}

Tabla VI

Resultados obtenidos por el grupo B del instituto de FP Torre Pacheco después de la aplicacín de la escala de actitudes hacia el Estudio de las Ciencias Experimentales.

\begin{tabular}{|cccccccccc|}
\hline fTEM & $N^{2}$ DE 1 & $N^{2}$ DE 2 & $N^{2}$ DE 3 & $N^{2}$ DE 4 & $N^{2}$ DE 5 & TOTAL & $\%$ & TOTAL (+o-) & $\%$ \\
\hline 1 & 4 & 14 & 6 & 0 & 0 & 50 & 27 & -22 & -46 \\
2 & 2 & 11 & 3 & 8 & 0 & 65 & 43 & -7 & -15 \\
3 & 1 & 6 & 13 & 4 & 0 & 68 & 46 & -4 & -8 \\
4 & 5 & 12 & 3 & 3 & 1 & 55 & 32 & -17 & -35 \\
5 & 9 & 11 & 4 & 0 & 0 & 43 & 20 & 29 & -60 \\
6 & 7 & 9 & 1 & 5 & 2 & 58 & 35 & -14 & -29 \\
7 & 1 & 4 & 6 & 8 & 5 & 84 & 63 & +12 & +25 \\
8 & 8 & 6 & 9 & 1 & 0 & 51 & 28 & -21 & -44 \\
9 & 2 & 11 & 8 & 3 & 0 & 60 & 38 & -12 & -25 \\
10 & 2 & 9 & 9 & 4 & 0 & 63 & 41 & -9 & -19 \\
11 & 6 & 13 & 2 & 3 & 0 & 50 & 27 & -22 & -46 \\
12 & 10 & 9 & 4 & 1 & 0 & 44 & 21 & -28 & -58 \\
13 & 0 & 4 & 6 & 9 & 5 & 87 & 66 & +15 & +31 \\
TOTAL & 58 & 119 & 74 & 49 & 13 & 778 & 37 & 158 & -25 \\
\hline
\end{tabular}

